Sol vent effects on el ectroni c structure of act i ve si te of azuri $n$ by pol arizable cont i nuum model

\begin{tabular}{|c|c|}
\hline 著者 & $\begin{array}{l}\text { Sugi mor i Ki mi kazu, Shuku Tomf umi, Sugi yama } \\
\text { Ayume, Nagao H dem, Sakur ai Takeshi, } \\
\text { N shi kawa Ki yoshi }\end{array}$ \\
\hline 著者別表示 & 杉森 公一, 長尾 秀実, 櫻井 武, 西川 清 \\
\hline $\begin{array}{l}\text { journal or } \\
\text { publ i cat } i \text { on title }\end{array}$ & Pol yhedr on \\
\hline vol une & 24 \\
\hline nunber & $16-17$ \\
\hline page $r$ ange & 2671- 2675 \\
\hline year & $2015-11-17$ \\
\hline URL & ht t p: //doi . or g/10. 24517/00030455 \\
\hline
\end{tabular}




\title{
Solvent Effects on Electronic Structure of Active Site of Azurin by Polarizable Continuum Model
}

\author{
Kimikazu Sugimori ${ }^{a}$, Tomofumi Shuku ${ }^{a}$, Ayumu Sugiyama ${ }^{a}$, \\ Hidemi Nagao $^{a}$, Takeshi Sakurai ${ }^{b}$, Kiyoshi Nishikawa ${ }^{a}$ \\ ${ }^{a}$ Division of Mathematical and Physical Science, Graduate School of Natural \\ Science and Technology, Kanazawa University, Kakuma, Kanazawa 920-1192, \\ Japan \\ ${ }^{\mathrm{b}}$ Division of Material Chemistry, Graduate School of Natural Science and \\ Technology, Kanazawa University, Kakuma, Kanazawa, Ishikawa 920-1192, Japan
}

\begin{abstract}
We present a cluster model for the active site of oxidized azurin, and investigate the electronic structure of the active site of oxidized azurin by using density functional calculations with polarizable continuum model. The singly occupied molecular orbital (SOMO) and spin density in the model widely distribute around the $\mathrm{Cu} 3 d_{x^{2}-y^{2}}$ and $\mathrm{S}(\mathrm{Cys} 112) 3 p$ orbitals. The dependency of electronic properties such as partial charge density and spin density on the dielectric constant is discussed. We find that partial spin density and charge density on the copper ion become larger, when the dielectric constant increases.
\end{abstract}

Key words: blue copper protein, azurin, DFT calculation, polarizable continuum model 


\section{Introduction}

Azurin is one of blue copper proteins with function of the electron transfer. The active site of azurin consists of a copper ion and five residues, which are His46, Cys112, His117, Met121, and Gly45. The copper ion is strongly connected to His46, Cys112, and His117 with coordination bonds and is weakly bonding to other residues, and the structure around the copper ion is a distorted trigonal bipyramid. The active site of blue copper proteins is generally characterized by an intense absorption at about $600 \mathrm{~nm}$ (blue color), an electron paramagnetic resonance spectrum with narrow hyperfine splittings, and a high reduction potential[1].

Solomon and co-workers [2-5] have summarized functions of blue copper proteins and have investigated electronic structures of the active sites in the proteins by quantum chemical calculations with computational models. For these metalloproteins, the metal ion and its environment are important from the viewpoint of catalysis in active sites. The functions of metalloproteins are summarized in Ref.[5]; (1) allosteric interactions between the active sites in different subunits, (2) organization of metal sites within a protein molecule for directional electron transfer (ET) to a catalytic site, (3) surface recognition sites for interactions with donor and acceptor proteins, (4) covalent ET pathways within the protein, (5) forming substrate access channels to the active site, (6) providing an active site hydrophobic environment within the protein in aqueous solution, (7) forming a substrate binding pocket near the metal active site, (8) assembling additional reactive covalently or non covalently bound organic cofactors for catalysis, (9) providing specific charge and hydrogen-bonding residues near the metal site to assist in catalysis, and (10) imposing a unique geometry on the metal site.

Recently, the blue copper proteins including azurin have been investigated experimentally and theoretically by many groups[1,6-12]. The geometry of the active site of azurin has been estimated by the density functional theory (DFT) with B3LYP method[7] and quantum mechanics/molecular mechanics (QM/MM) method[8]. The hyperfine coupling constants in the active site have been calculated by DFT with B1LYP[9] and B3LYP[10] methods, and also by $\mathrm{QM} / \mathrm{MM}$ approach[11]. The electronic structure and the $g$-tensor of the active site of azurin have been investigated by ab initio multireference determinantal configuration interaction (MRD-CI) calculations[12]. 
Solvent effects on electronic structure of molecules have been investigated by many chemists and physicists to understand molecular structure, mechanism of chemical reactions in solution, etc. by using quantum-chemical calculations and molecular dynamics simulations. Physical properties such as geometry of molecules and charge distribution in solution often vary from those in vacuum[13]. Ryde et al. [7] have also performed a geometry optimization of the active site of blue copper proteins including azurin by quantum-chemical calculations with polarizable continuum model (PCM)[14]. Their results suggest that weak axial bond of $\mathrm{Cu}-\mathrm{S}($ Met121) in solution is more elongated than that in vacuum.

In this study, we investigate solvent effects on electronic structure of active site of oxidized azurin by density functional calculations. A cluster model for the active site of oxidized azurin is presented, and we calculate physical properties such as charge density and spin density of the model cluster involving solvent effects. We also present the dependence of those properties on the dielectric constant. Finally, we discuss the charge or electron transfer in the active site of azurin in relation to solvent effect.

\section{Computational method}

We present a cluster model for the active site of oxidized azurin and methods used in this study.

\section{2-1. Computational model for active site}

We consider a cluster model, which consists of a copper ion and five residues as shown Fig.1, for the active site of oxidized azurin. In the configuration of the cluster model, we use the coordinate of atoms around the active site of Pseudomonas aeruginosa azurin (code 4AZU) in Protein Data Bank, which is determined by X-ray diffraction results at $\mathrm{pH}$ 5.5[6]. The configuration of all hydrogen atoms in the cluster model is determined by Hartree-Fock/3-21G* geometry optimization with fixed configuration of other all atoms. Ligand distances, angles, and dihedral angles of the cluster model used in this study are summarized in Table 1. In the calculations of this study, we use a condition that the total charge of the cluster model is +1 . 
2-2. Solvent effect using polarizable continuum model

We perform density functional calculations with Becke's three-parameter hybrid exchange functional[15] and the Lee-Yang-Parr correlation functional[16] (B3LYP) for the cluster model as shown in Fig. 1 by Gaussian03 program package[17]. We calculate partial charge and partial spin density on each atom by Mulliken population analysis (MPA) and natural population analysis (NPA)[18] with 6-31G* split-valence basis set[19].

For investigation of electronic structure of the active site of oxidized azurin in solution, we use polarizable continuum model (PCM)[14] in DFT calculations. An integral equation formulation model (IEF-PCM) [20] is adopted. In present calculations, values of dielectronic constant $\epsilon_{r}$ are arbitrarily selceted in the region from 2 to 78 .

3. Results and discussion

3-1. Spin density and charge density in vacuum

Figure 2(a) shows the singly occupied molecular orbital (SOMO) of the cluster model by unrestricted B3LYP/6-31G* calculation. We can find $3 \mathrm{~d}_{x^{2}-y^{2}}$ orbital $\pi$ antibonded to the sulfur atom in the Cys112 and $\sigma$ antibonded to the nitrogen atoms in His46 and His117. SOMO distributes on the nitrogen atoms in His46 and His117, and is not much on other atoms in His46 and His117. On the other hand, we can find that the distribution of SOMO widely lies over Cys112. SOMO largely distributes on the sulfur atom, two $\mathrm{H}$ atoms in $\mathrm{C}_{\beta} \mathrm{H}_{2}$ in Cys112, and the nitrogen atom in backbone of Cys112. We also find small distribution of SOMO around O atom in Gly45. Figure 2(b) shows the spin density of the cluster model. The spin density distributes around copper ion, $\mathrm{S}($ Cys112), N(His46) and N(His117) atoms, and we also find small distribution of spin density on the $\mathrm{H}$ atoms in $\mathrm{C}_{\beta} \mathrm{H}_{2}$ and the nitrogen atom in backbone of Cys112. In three coordination bonds to copper ion, SOMO widely distributes to only the bonding direction to the side of Cys112. These results suggest that histidine or imidazole has a role of restraint of spatial distribution for the direction of the coordination-bonding of the nitrogen atom to the copper ion. 
Table 2 summarizes the atomic charge and spin density of the cluster model in vacuum by Mulliken population analysis(MPA) and natural population analysis(NPA). From partial spin density by MPA in Table 2, we find the largest value about $53.2 \%$ on the copper ion and the second largest value about $38.8 \%$ on the sulfur ion in Cys112. From NPA, we find that the valence of the copper ion becomes 1.26. Atomic charges and Mulliken spin densities of the similar cluster model to the present model in vacuum have been presented in Ref.[12].

3-2. Spin density and atomic charge in solution

Tables 3 and 4 show the spin densities and the charge distribution for various dielectric constants, respectively. The dielectric constants $\epsilon_{r}$ is varied from 2 to 78. In general, $\epsilon_{r}=78$ corresponds to water molecule. We find asymptotical changes of spin density and atomic charge between the copper ion and the sulfur ion in Cys112. As increasing $\epsilon_{r}$, the spin density of $\mathrm{Cu}$ ion becomes increasing, and the spin density of $\mathrm{S}_{\gamma}(\mathrm{Cys} 112)$ is decreasing. On the other hand, the atomic charge of $\mathrm{Cu}$ ion increases from 0.644 to 0.681 , and the atomic charge of $\mathrm{S}_{\gamma}($ Cys112) decreases from -0.160 to -0.233 . These changes of atomic charge and spin densities suggest that electron with $\beta$ spin on copper ion slightly moves to the sulfur ion in Cys112 in the case of increasing the dielectric constant. From this electron transfer, the spin density of the copper ion becomes larger than that in vacuum, and the charge distribution also becomes larger.

\section{3-3. A simple model for solvent effect}

In this subsection, we briefly discuss the solvent effects of electronic properties presented in previous subsection by using a simple Hubbard model[21-23]. In this study, we focus $d_{x^{2}-y^{2}}$ orbital of the copper ion and $p_{y}$ orbital of sulfur ion in Cys112 in the active site of azurin. We assume on-site repulsion $U$ on only copper site, because it is well known that the on-site coulomb repulsion $U$ of the copper ion is large (about $8 \mathrm{eV}$ ). Therefore, for the most simple case, we can consider a 2-site system with 3 electrons as a simple model for the active site. By solving electronic structure of this simple model, we can find that partial charge of $d$-orbital becomes larger than that of $p$-orbital, when $t / U \gg 1$, and that partial spin of $d$-orbital becomes smaller. On the other hand, in the case 
of $t / U \ll 1$, partial charge on $d$-orbital becomes smaller, and partial spin on $d$-orbital becomes larger, because of the large on-site repulsion. In the case of the active site of oxidized azurin, we can expect that the transfer integral becomes smaller than the on-site repulsion on the copper ion in the active site.

Next, we consider a simple model for solvent effects. We assume the effective electron-electron interaction $W$ between sites in the system and solvent molecules. The effective interaction is clearly related to the polarization of the solvent molecules. If solvent molecules have large dielectric constant, the effective interaction $W$ becomes large. In the frame work of a Random Phase Approximation[24,25], the effective on-site coulomb potential $U_{\text {eff }}$ on the $d$ orbital can be written as

$$
U_{\text {eff }}=\frac{U}{1-U^{2} W^{2}}
$$

From Eq.(1), we find that the effective interaction $U_{\text {eff }}$ becomes larger than the bare interaction $U$, when the dielectric constant is larger than that in vacuum. Therefore, from Eq.(1), we can expect that spin density of copper ion in the active site of oxidized azurin in solution with large dielectric constant becomes larger than that in vacuum. This effect arises from the strong electron correlation on the copper ion. Since on-site coulomb interactions of $p$ orbitals are small, the solvent effects of electronic correlation in $p$ orbital might be small.

\section{Concluding remarks}

In this study, we have presented a computational model for the active site of oxidized azurin and have investigated the electronic structure of the active site model by using the density functional methods with polarizable continuum model. The partial atomic charge and spin density on some ions have been calculated for various dielectric constants. We have presented a simple model for the active site of oxidized azurin by a Hubbard model, and have discussed the dependence of the charge and spin distributions on the dielectric constants.

For the cluster model in vacuum, the distribution of SOMO and the spin density widely lie over Cys112. The distribution of them over histidines is not so much. In solution, the partial spin and charge of the copper ion become 
larger than that in vacuum, when the dielectric constant is larger. The spin and charge of sulfur ion in Cys112 become smaller. These results suggest that electron with $\beta$ spin moves from the copper ion to the sulfur ion, if the dielectric constant becomes larger. We have found the electron moving because of the strong electron correlation on the copper ion by using a Hubbard model.

Since the active site of azurin is surrounded by many residues, the electronic structure of active site of azurin is weakly interacted with solvent molecules. We need to consider larger systems and to use other excellent computational methods such as QM/MM method. The contribution to the electronic structure of the active site of azurin from neighbor residues around the active site will be presented elsewhere. Electronic structure of another cluster model terminated by $\mathrm{H}$ atoms for $\mathrm{N}$ - and $\mathrm{C}$ - termini in each residue in the cluster model presented in this study will be also presented elsewhere. The electronic structure of the new model also becomes the same tendency to the present model.

H.N is grateful for a financial support of the Ministry of Education, Science and Culture of Japan (Research No.15550010).

\section{References}

[1] E. T. Adman, Adv. Protein Chem. 42 (1991) 145.

[2] K. W. Penfield, A. A. Gewirth, E. I. Solomon, J. Am. Chem. Soc. 107 (1985) 4519-4529.

[3] E. I. Solomon, M. J. Baldwin, M. D. Lowery, Chem. Rev. 92 (1992) 521-542.

[4] J. A. Guckert, M. D. Lowery, E. I. Solomon, J. Am. Chem. Soc. 117 (1995) $2817-2844$.

[5] E. I. Solomon, R. K. Szilagyi, S. D. George, L. Basumallick, Chem. Rev. 104 (2004) 419-458.

[6] H. Nar, A. Messerschmidt, R. Hunber, M. van de Kamp, G. W. Canters, J. Mol. Biol. 221 (1991) 765-772.

[7] U. Ryde, M. H. M. Ollson, B. O. Roos, A. C. Borin, Theor. Chem. Acc. 105 (2001) 452-462. 
[8] U. Ryde, M. H. M. Olsson, Int. J. Quantum Chem. 81 (2001) 335-347.

[9] A. R. Jaszewski, J. Jezierska, Chem. Phys. Lett. 343 (2001) 571-580.

[10] M. Swart, Density functional theory applied to copper proteins, Ph.D. thesis, Rijkuniversiteit Groningen, Groningen (2002).

[11] S. Moon, S. Patchkovskii, D. R. Salahub, J. Mol. Struct. (Theochem) 632 (2003) 287-295.

[12] M. van Gastel, J. W. A. Coremans, H. Sommerdijk, M. C. van Hemert, E. J. J. Groenen, J. Am. Chem. Soc. 124 (2002) 2035-2041.

[13] M. L. J. Drummond, Prog. Biophys. moledc. Biol. 47 (1986) 1-29.

[14] S. Miertuš, E. Scrocco, J. Tomasi, Chem. Phys. 55 (1981) 117.

[15] A. D. Becke, J. Chem. Phys. 98 (1993) 5648.

[16] C. Lee, W. Yang, R. G. Parr, Phys. Rev. B 37 (1988) 785.

[17] M. J. Frisch, G. W. Trucks, H. B. Schlegel, G. E. Scuseria, M. A. Robb, J. R. Cheeseman, J. A. Montgomery, Jr., T. Vreven, K. N. Kudin, J. C. Burant, J. M. Millam, S. S. Iyengar, J. Tomasi, V. Barone, B. Mennucci, M. Cossi, G. Scalmani, N. Rega, G. A. Petersson, H. Nakatsuji, M. Hada, M. Ehara, K. Toyota, R. Fukuda, J. Hasegawa, M. Ishida, T. Nakajima, Y. Honda, O. Kitao, H. Nakai, M. Klene, X. Li, J. E. Knox, H. P. Hratchian, J. B. Cross, C. Adamo, J. Jaramillo, R. Gomperts, R. E. Stratmann, O. Yazyev, A. J. Austin, R. Cammi, C. Pomelli, J. W. Ochterski, P. Y. Ayala, K. Morokuma, G. A. Voth, P. Salvador, J. J. Dannenberg, V. G. Zakrzewski, S. Dapprich, A. D. Daniels, M. C. Strain, O. Farkas, D. K. Malick, A. D. Rabuck, K. Raghavachari, J. B. Foresman, J. V. Ortiz, Q. Cui, A. G. Baboul, S. Clifford, J. Cioslowski, B. B. Stefanov, G. Liu, A. Liashenko, P. Piskorz, I. Komaromi, R. L. Martin, D. J. Fox, T. Keith, M. A. Al-Laham, C. Y. Peng, A. Nanayakkara, M. Challacombe, P. M. W. Gill, B. Johnson, W. Chen, M. W. Wong, C. Gonzalez, J. A. Pople, Gaussian 03, Revision B.05, Gaussian, Inc., Pittsburgh, PA, 2003.

[18] A. E. Reed, R. B. Weinstock, F. Weinhold, J. Chem. Phys. 83 (1985) 735.

[19] W. J. Hehre, L. Radom, P. v. R. Schleyer, J. A. Pople, Ab initio Molecular Orbital Theory, Wiley-Interscience, New York, 1986.

[20] E. Cancès, B. Mennucci, J. Tomasi, J. Chem. Phys. 107 (1997) 3032-3041.

[21] J. Hubbard, Proc. Roy. Soc. (London) A281 (1961) 401.

[22] J. Hubbard, Proc. Roy. Soc. (London) A276 (1963) 238.

[23] J. Hubbard, Proc. Roy. Soc. (London) A277 (1964) 237.

[24] D. Bohm, D. Pines, Phys. Rev. 92 (1953) 609.

[25] D. Pines, Phys. Rev. 92 (1953) 626. 
Table 1

Ligand distances, angles, and dihedral angles of active site model used in this study.

\begin{tabular}{|c|c|c|}
\hline & & parameter \\
\hline \multirow[t]{5}{*}{ Distance $(\AA)$} & $\mathrm{Cu}-\mathrm{O}(\mathrm{Gly} 45)$ & 2.955 \\
\hline & $\mathrm{Cu}-\mathrm{N}(\mathrm{His} 46)$ & 2.064 \\
\hline & $\mathrm{Cu}-\mathrm{S}(\mathrm{Cys} 112)$ & 2.267 \\
\hline & $\mathrm{Cu}-\mathrm{N}(\mathrm{His} 117)$ & 1.978 \\
\hline & $\mathrm{Cu}-\mathrm{S}(\mathrm{Met} 121)$ & 3.164 \\
\hline \multirow[t]{4}{*}{ Angle (deg) } & $\mathrm{N}($ His117)-Cu-N(His46) & 108.3 \\
\hline & $\mathrm{S}(\mathrm{Cys} 112)-\mathrm{Cu}-\mathrm{N}(\mathrm{His} 117)$ & 123.7 \\
\hline & $\mathrm{S}(\mathrm{Cys} 112)-\mathrm{Cu}-\mathrm{S}(\mathrm{Met} 121)$ & 110.5 \\
\hline & $\mathrm{S}($ Cys112)-Cu-O(Gly45) & 100.3 \\
\hline \multirow[t]{5}{*}{ Dihedral angle (deg) } & $\mathrm{N}(\mathrm{His} 117)-\mathrm{Cu}-\mathrm{S}(\mathrm{Cys} 112)-\mathrm{C}_{\beta}$ & 91.6 \\
\hline & $\mathrm{S}\left(\right.$ Cys112)-Cu-N(His46)- $\mathrm{C}_{\gamma}$ & -13.6 \\
\hline & $\mathrm{S}(\mathrm{Cys} 112)-\mathrm{Cu}-\mathrm{N}(\mathrm{His} 117)-\mathrm{C}_{\gamma}$ & -40.7 \\
\hline & $\mathrm{S}\left(\right.$ Cys112)-Cu-S(Met121)- $\mathrm{C}_{\gamma}$ & 46.9 \\
\hline & $\mathrm{S}(\mathrm{Cys} 112)-\mathrm{Cu}-\mathrm{O}(\mathrm{Gly} 45)-\mathrm{C}$ & 82.8 \\
\hline
\end{tabular}


Table 2

Partial atomic charges and partial spin densities on each atom by Mulliken Population Analysis(MPA) and Natural Population Analysis(NPA).

\begin{tabular}{|c|c|c|c|c|}
\hline \multirow[b]{2}{*}{ Residue } & \multirow[b]{2}{*}{ Atom } & \multicolumn{2}{|c|}{ MPA } & \multirow{2}{*}{$\frac{\mathrm{NPA}}{\text { atomic charge(a.u.) }}$} \\
\hline & & atomic charge(a.u.) & spin density(\%) & \\
\hline \multirow{4}{*}{ Cys112 } & $\mathrm{Cu}$ & 0.6448 & 53.227 & 1.2605 \\
\hline & $\mathrm{C}_{\alpha}$ & -0.0080 & -0.007 & -0.1594 \\
\hline & $\mathrm{C}_{\beta}$ & -0.4464 & -1.520 & -0.5852 \\
\hline & $\mathrm{S}_{\gamma}$ & -0.1603 & 38.769 & -0.3691 \\
\hline \multirow[t]{5}{*}{ His46 } & $\mathrm{C}_{\gamma}$ & 0.2931 & -0.052 & 0.1339 \\
\hline & $\mathrm{N}_{\delta 1}$ & -0.5662 & 2.415 & -0.6495 \\
\hline & $\mathrm{C}_{\delta 2}$ & 0.2263 & -0.301 & 0.1956 \\
\hline & $\mathrm{C}_{\epsilon 1}$ & -0.5139 & 0.025 & -0.5038 \\
\hline & $\mathrm{N}_{\epsilon 2}$ & -0.0195 & 0.268 & -0.0798 \\
\hline \multirow[t]{5}{*}{ His117 } & $\mathrm{C}_{\gamma}$ & 0.2728 & -0.126 & 0.1460 \\
\hline & $\mathrm{N}_{\delta 1}$ & -0.5264 & 3.601 & -0.6627 \\
\hline & $\mathrm{C}_{\delta 2}$ & 0.2308 & -0.424 & 0.1899 \\
\hline & $\mathrm{C}_{\epsilon 1}$ & 0.0138 & 0.341 & -0.0742 \\
\hline & $\mathrm{N}_{\epsilon 2}$ & -0.5239 & 0.047 & -0.5012 \\
\hline \multirow[t]{3}{*}{ Gly 45} & $\mathrm{C}$ & 0.5979 & -0.015 & 0.6871 \\
\hline & $\mathrm{O}$ & -0.5303 & 0.022 & -0.7012 \\
\hline & $\mathrm{C}_{\alpha}$ & -0.2391 & -0.001 & -0.3368 \\
\hline \multirow[t]{3}{*}{ Met121 } & $\mathrm{C}_{\gamma}$ & 0.1987 & -0.023 & -0.5907 \\
\hline & $\mathrm{S}_{\delta}$ & 0.0769 & -0.051 & 0.1822 \\
\hline & $\mathrm{C}_{\epsilon}$ & -0.1603 & 0.005 & -0.5678 \\
\hline
\end{tabular}


Table 3

Dependency of spin density (\%) on dielectric constant by MPA.

\begin{tabular}{lcccccccc}
\hline \hline \multicolumn{1}{c}{$\epsilon_{r}$} & vacuum & 2 & 4 & 8 & 16 & 24 & 32 & 78 \\
\hline $\mathrm{Cu}$ & 53.227 & 55.803 & 57.630 & 58.788 & 59.462 & 59.704 & 59.830 & 60.059 \\
$\mathrm{~S}_{\gamma}($ Cys112) & 38.769 & 36.151 & 34.127 & 32.793 & 32.002 & 31.714 & 31.565 & 31.290 \\
$\mathrm{C}_{\beta}($ Cys112) & -1.520 & -1.437 & -1.370 & -1.324 & -1.297 & -1.287 & -1.282 & -1.272 \\
$\mathrm{~N}_{\delta 1}($ His117) & 3.601 & 3.871 & 4.074 & 4.206 & 4.283 & 4.312 & 4.326 & 4.353 \\
$\mathrm{O}($ Gly45) & 0.022 & 0.005 & 0.004 & 0.003 & 0.003 & 0.003 & 0.002 & 0.002 \\
$\mathrm{~S}_{\delta}$ (Met121) & -0.051 & -0.057 & -0.061 & -0.064 & -0.065 & -0.066 & -0.066 & -0.067 \\
\hline \hline
\end{tabular}


Table 4

Dependency of atomic charge (a.u.) on dielectric constant by MPA.

\begin{tabular}{lrccccccc}
\hline \hline \multicolumn{1}{c}{$\epsilon_{r}$} & vacuum & 2 & 4 & 8 & 16 & 24 & 32 & 78 \\
\hline $\mathrm{Cu}$ & 0.644 & 0.660 & 0.670 & 0.676 & 0.679 & 0.680 & 0.680 & 0.681 \\
$\mathrm{~S}_{\gamma}($ Cys112) & -0.160 & -0.185 & -0.205 & -0.212 & -0.228 & -0.231 & -0.233 & -0.236 \\
$\mathrm{C}_{\beta}($ Cys112) & -0.446 & -0.444 & -0.442 & -0.440 & -0.439 & -0.439 & -0.439 & -0.439 \\
$\mathrm{~N}_{\delta 1}($ His117) & -0.526 & -0.580 & -0.583 & -0.585 & -0.586 & -0.587 & -0.587 & -0.587 \\
$\mathrm{O}($ Gly45) & -0.530 & -0.414 & -0.429 & -0.438 & -0.443 & -0.448 & -0.446 & -0.447 \\
$\mathrm{~S}_{\delta}$ (Met121) & 0.077 & 0.076 & 0.075 & 0.075 & 0.074 & 0.074 & 0.074 & 0.074 \\
\hline \hline
\end{tabular}




\section{Figure captions}

Figure 1 Cluster model for active site of oxidized azrin.

Figure 2 SOMO and spin density of the cluster model. (a)Distribution of SOMO (isovalue $=0.02$ a.u., and orbital energy $=-9.1931 \mathrm{eV}$ ). (b)Distribution of spin density (isovalue $=0.0012$ a.u.). 


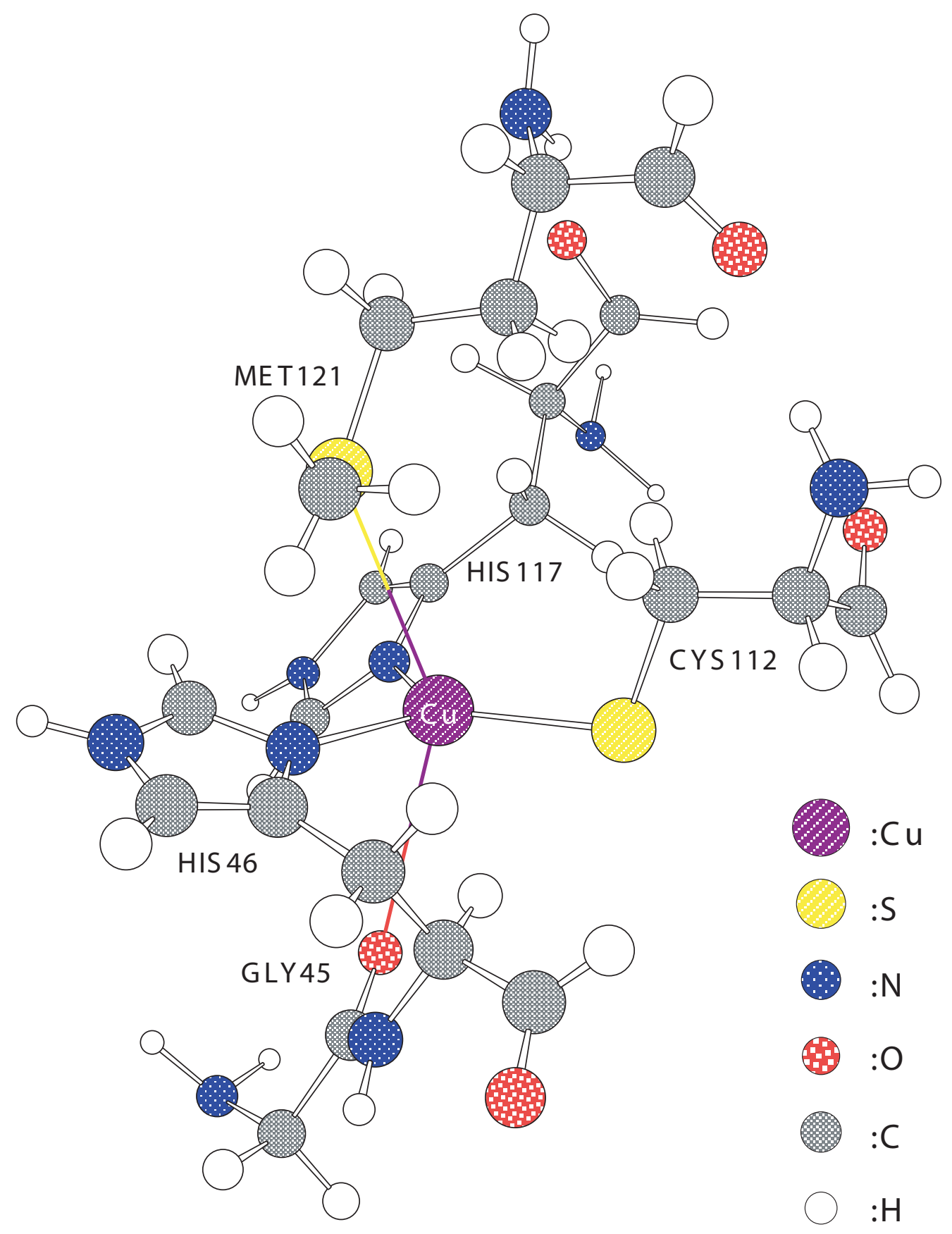

Figure $1 \mathrm{~K}$. Sugimori et al. 

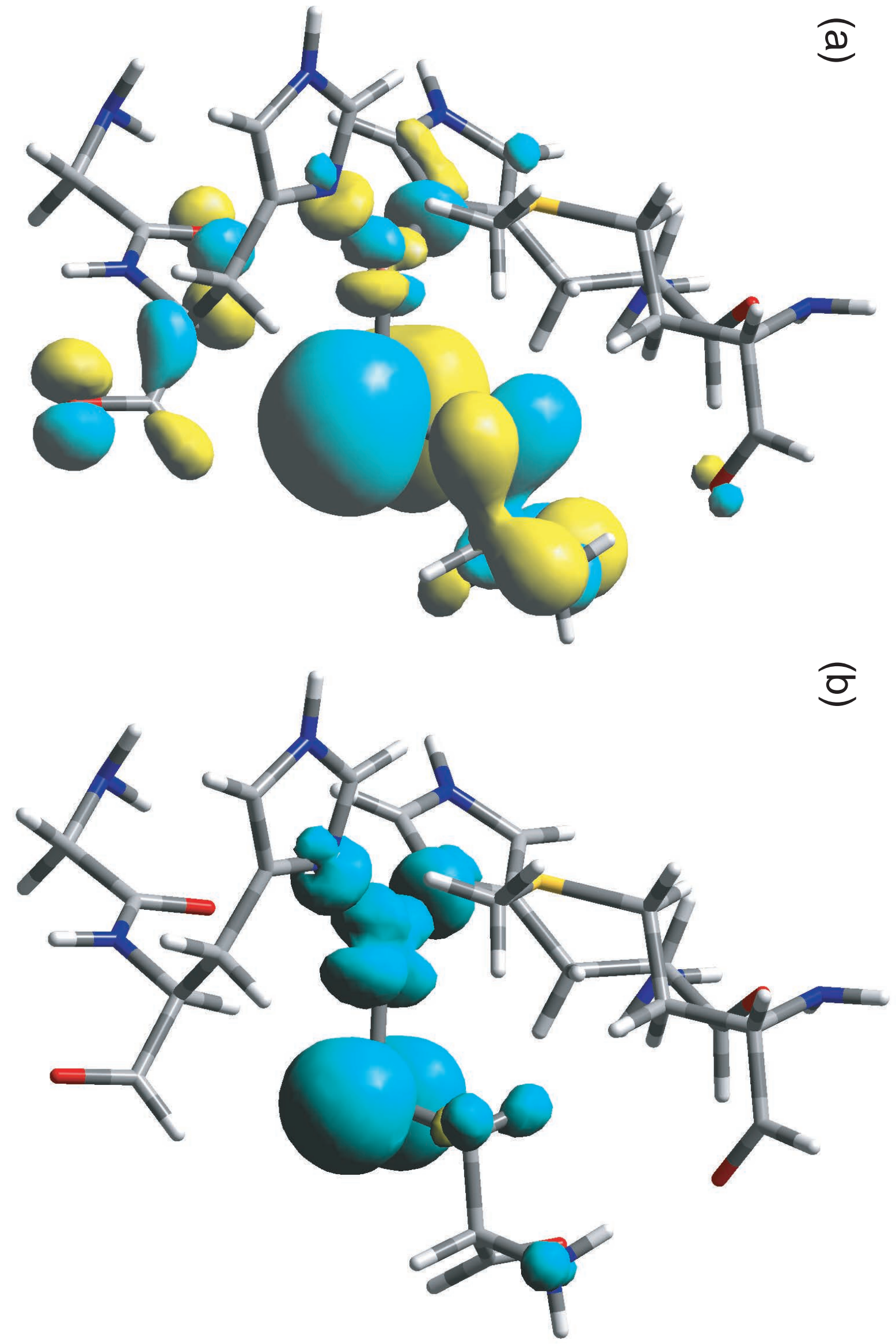

Figure 2 K. Sugimori et al. 